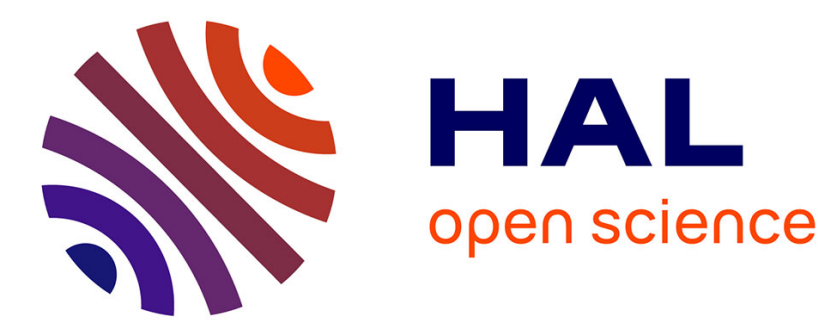

\title{
MR spectroscopy of normal and impaired fetal brain
} Angèle Viola, Yann Le Fur, Patrick Viout, Nadine Girard

\section{To cite this version:}

Angèle Viola, Yann Le Fur, Patrick Viout, Nadine Girard. MR spectroscopy of normal and impaired fetal brain. European Biophysical Societies (eds) Roberts G., Watts A. Encyclopedia of Biophysics., Springer, 2021, 10.1007/978-3-642-35943-9_10099-1 . hal-03451017

\section{HAL Id: hal-03451017 https://hal.science/hal-03451017}

Submitted on 26 Nov 2021

HAL is a multi-disciplinary open access archive for the deposit and dissemination of scientific research documents, whether they are published or not. The documents may come from teaching and research institutions in France or abroad, or from public or private research centers.
L'archive ouverte pluridisciplinaire HAL, est destinée au dépôt et à la diffusion de documents scientifiques de niveau recherche, publiés ou non, émanant des établissements d'enseignement et de recherche français ou étrangers, des laboratoires publics ou privés. 


\section{MR spectroscopy of normal and impaired fetal brain}

Angèle Viola ${ }^{1}$, Yann le Fur ${ }^{1}$, Patrick Viout ${ }^{1}$, Nadine Girard ${ }^{1-2}$

1. CNRS, Aix-Marseille Univ, Centre de Résonance Magnétique Biologique et Médicale, CRMBM UMR CNRS 7339, Faculté des Sciences Médicales et Paramédicales la Timone, Marseille, FRANCE

2. Service de Neuroradiologie Diagnostique et Interventionnelle, Hôpital la Timone, APHM, Marseille, FRANCE

\section{Email addresses:}

angele.viola@univ-amu.fr

yann.le-fur@univ-amu.fr

patrick.viout@ap-hm.fr

nadine.girard@ap-hm.fr

\section{Synonyms:}

In vivo Nuclear Magnetic Resonance (NMR) 


\section{Introduction}

Magnetic Resonance Spectroscopy (MRS) is a safe, non-invasive and quantitative method used in the research and clinical settings to study the metabolic basis of physiological and pathological processes in tissues. MRS techniques are available on clinical MRI systems and can be performed during a standard scanning protocol following the acquisition of anatomical images. Brain MRS has a number of applications in neuroradiology including pediatric neuroradiology (e.g. tumors, inborn errors of metabolism, anomalies of brain development...). MRS can also be performed during the pregnancy to assess fetal brain metabolism. Indications of fetal brain MRI (e.g. abnormal ultrasound scan such as ventricular dilatation and suspicion of brain malformation, pregnancy with a risk of brain damage as in cytomegalovirus seroconversion, intrauterine growth retardation, twin to twin transfusion syndrome in multiple pregnancy, and family history of genetic disease and of brain malformation among the siblings) represent an opportunity to study the neurochemical correlates of brain development and their alterations using MRS. Since the beginning of fetal brain explorations with MRS in the early 90's (Heerschap and van den Berg, 1994), several studies have confirmed the unequaled ability of MRS for monitoring neurochemical changes related to normal or impaired brain development and have improved the understanding of cerebral metabolic maturation.

\section{Proton MRS}

Among the atomic nuclei with magnetic properties (non-zero spin quantum number $I$ ) relevant for in vivo brain MRS, proton $\left({ }^{1} \mathrm{H}\right)$ is the most widely studied because it possesses the largest gyromagnetic ratio $(\gamma)$, the highest sensitivity (signal to noise per unit of time which is dependent on the magnetic field B0 and $\gamma$ ), and its natural abundance is close to $100 \%$ (Table 1). 
In vivo MRS allows detection of mobile metabolites whose concentration is at least in the $\mathrm{mM}$ range because of the poor sensitivity of NMR. A wide range of metabolite signals can be detected in the proton brain spectrum such as $N$-acetylaspartate (NAA), a marker of neurons in the adult brain which is also present in oligodendrocyte precursors in the developing brain, aminoacids including neurotransmitters (e.g. glutamate, aspartate, taurine, glycine), polyols (myo-inositol, scyllo-inositol), $\gamma$-aminobutyrate (GABA), lactate, fatty acids, creatine $(\mathrm{Cr})$ and phosphocreatine $(\mathrm{PCr})$, choline containing compounds (mostly glycerophosphocholine and phosphocholine) among others (Figure 1). In addition, the signals of macromolecules can be detected and quantified with advanced post-processing methods. Metabolites and macromolecules concentrations vary across brain regions and structures, as well as with physiological processes (e.g. brain development, maturation, and ageing). Pathological processes underlying disease development can also significantly modify brain metabolic profile. MRS is arguably the primary method to non-invasively follow changes in brain neurochemistry as well as to provide metabolic markers with diagnostic value.

Table I: Characteristics of magnetic nuclei of interest for in vivo MRS

\begin{tabular}{|c|c|c|c|c|}
\hline Nucleus & Spin & $\gamma\left(\mathbf{1 0}^{\mathbf{7}} \mathbf{T}^{-1} \mathbf{S}^{-1}\right)$ & $\begin{array}{c}\boldsymbol{v}_{\mathbf{0}} \text { in MHz at } \\
\mathbf{1 . 5 T}(\mathbf{a}) \text { and } \\
\mathbf{3 T}(\mathbf{b})\end{array}$ & $\begin{array}{c}\text { Natural } \\
\text { abundance } \\
(\%)\end{array}$ \\
\hline${ }^{1} \mathrm{H}$ & $1 / 2$ & 26.75 & $\begin{array}{c}(\mathrm{a}): 64 \\
(\mathrm{~b}): 128\end{array}$ & 99.98 \\
\hline${ }^{31} \mathrm{P}$ & $1 / 2$ & 10.84 & $(\mathrm{a}): 26$ & 100 \\
& & & $(\mathrm{~b}): 52$ & \\
\hline${ }^{13} \mathrm{C}$ & $1 / 2$ & 6.73 & $(\mathrm{a}): 16$ & 1.1 \\
& & & $(\mathrm{~b}): 32$ & \\
\hline
\end{tabular}

$v_{0}$ : frequency resonance given by the resonance condition $\left(v_{0}=\gamma \mathrm{B} 0 / 2 \pi\right)$ 


\section{(Figure 1)}

\section{Features of the MR spectrum}

MR spectra contain information on the metabolites conveyed by the chemical shift, the spinspin coupling (or $J$-coupling) and the area of the resonance peaks.

Chemical shift: although the resonance frequency of a given nucleus is determined by the resonance condition $\left(v_{0}=\gamma \mathrm{B} 0 / 2 \pi\right)$, distinct resonance peaks are usually present in the MRS spectrum. Indeed, nuclei (protons) borne by a same molecule do not resonate at the same frequency because they generally experience different local magnetic fields owing to differences in chemical environment. This effect termed as "chemical shift" can be measured with a scale in $\mathrm{Hz}$ or in part per million (ppm). The scale in ppm has the advantage of being independent of B0, which allows comparison between spectra obtained at different magnetic field strengths.

J-coupling (spin-spin coupling or scalar coupling): this magnetic interaction occurs through the electrons involved in chemical bonds between neighboring nuclei. The strength of the coupling decreases beyond three bonds. $J$-coupling leads to the splitting of peaks in multiple components with characteristic spacings and intensities (e.g. doublets, triplets etc..). This interaction is measured by a coupling constant termed $J$ usually expressed in Hz. The observation of peak multiplicity requires narrow linewidth which is difficult to achieve in fetal brain spectra acquired at $1.5 \mathrm{~T}$. Nevertheless, an interesting effect due to the evolution of $J$ coupled magnetization with $\mathrm{TE}$ can be observed at $1.5 \mathrm{~T}$ using the Point RESolved Spectroscopy (PRESS) sequence with respect to the lactate doublet generated by the interaction of methyl protons with the methine proton (Figure 1). At long TEs (e.g. 135 or $144 \mathrm{~ms}$ ), the doublet is inverted which allows unambiguous identification of the lactate signal usually hindered by the presence of overlapping lipid signals at short TE. Acquisitions with short TEs enable the detection of molecules having short transverse relaxation time $\left(\mathrm{T}_{2}\right)$ such as lipids and 
macromolecules, whereas acquisition with long TEs result in spectrum simplification that can be useful for quantification purpose in spectral regions with overlapping signals from different metabolites.

\section{Acquisition methods}

So far, proton MRS of the fetal brain has been carried out in specialized centers using clinical MR systems operating at $1.5 \mathrm{~T}$ or $3 \mathrm{~T}$ with coils suitable for pregnant women (anterior body phased-array coils containing several elements and posterior spinal coils). The acquisition needs to be fast (few minutes) to avoid signal loss and artefacts due to maternal and/or fetal movement especially in non-sedated women. An advantage of ${ }^{1} \mathrm{H}-\mathrm{MRS}$ is that this technique is performed using coils designed for MRI. Single voxel spectroscopy (SVS) is currently the dominant method in the field of fetal brain MRS. SVS is a robust technique allowing 3D spatial localization of the signal from a well-defined volume of interest (VOI) or voxel within a body region. Anatomical images, which are obtained prior to the MRS acquisition are used to guide the positioning of the voxel within the fetal brain. 3D-spatial volume selection is achieved by applying radiofrequency (RF) and gradients pulses to three orthogonal magnetization slices selecting thereby their intersection (cube-shape VOI) for signal localization. The main SVS sequences are the PRESS and the Stimulated Echo Acquisition Mode (STEAM) sequences (de Graaf, 2007). Although STEAM allows for shorter TEs, PRESS is the most applied technique in fetal brain MRS because the signal generated with PRESS is twice the intensity of that produced with STEAM (there is a signal loss of 50\% with the stimulated echo compared to the spin echo).

$\operatorname{PRESS}\left(90^{\circ}-180^{\circ}-180^{\circ}\right)$ : this sequence is based on a double spin echo (Figure 2). A $90^{\circ}$ excitation pulse is applied followed by two refocusing $180^{\circ}$ pulses. After the excitation pulse, magnetization is nutated into the transverse plane and loses coherence. The refocusing pulses 
are used to rephase magnetization components. The first $180^{\circ}$ pulse flip magnetization so that spins are refocused, a spin echo is formed corresponding to the intersection of two slices. After the second $180^{\circ}$ pulse, a spin echo localized to the VOI is formed at the end of a time called the time of echo (TE). Spoiling or crusher gradients are applied to destroy unwanted coherences. Acquisitions are repeated after a time interval called the time of repetition (TR). The number of repetitions (also termed averages, transients or scans) to get a fetal brain spectrum with sufficient signal-to-noise ratio (SNR) is around 100 averages depending on the time of echo. The spectra are acquired with or without water saturation.

\section{(Figure 2)}

\section{Water and lipid suppression}

Water is much more concentrated in brain (up to 50M) than metabolites which are present in the millimolar range. Consequently, the water signal can easily mask metabolite signals and cause baseline distortion. Therefore, water suppression methods such as frequency selective excitation are often applied to minimize water signal in brain spectra. In addition to water saturated spectra, non-saturated spectra can be acquired with the purpose of using the water signal as an internal reference for quantification. Another problem is the contamination from outside the VOI mainly due to lipid signals that can be eliminated by using outer volume suppression techniques.

\section{Assessment of spectrum quality}

The SNR and the full width at half maximum (FWHM) of the peaks are the main criteria to assess spectrum quality. Different types of artefacts can occur that will affect the quality of the spectra. Spectra showing artefacts due to maternal or fetal movement, Eddy currents, water saturation or outer volume contamination by subcutaneous fats should be discarded. 


\section{Data analysis}

Data are often processed by NMR spectroscopists using advanced methods and dedicated software implemented with metabolite and macromolecule databases containing simulated signals of metabolites. Data are processed either in the time domain (eg: AMARES, QUEST) or the frequency domain (eg LCmodel) (Figure 3). The analysis is usually semi quantitative, results are expressed as the ratio of two metabolite signals (Metabolite $1_{\text {signal }} /$ Metabolite $2_{\text {signal }}$ ) or the ratio of a metabolite signal to the sum of all metabolite signals (Metabolite $1_{\text {signal }}$ $\left./ \Sigma \mathrm{Metabolite}_{\text {signal }}\right)$, or the ratio of a metabolite signal to the water signal (Metabolite signal $/ \mathrm{H}_{2} \mathrm{O}_{\text {signal }}$ ) (the same thing applies to macromolecules) (Girard et al., 2006b). Absolute quantification requires the measurement of $\mathrm{T}_{1}$ and $\mathrm{T}_{2}$ relaxation times of each metabolite in normal and pathological brain parenchyma, as well as accurate assessment of water content in the VOI which requires segmentation of the brain tissue encompassed by the voxel in terms of gray matter, white matter, and ventricles on MRI. Relaxation time measurements are difficult to achieve in the fetal brain because repeated acquisitions with variable TE or TR are required, which results in long scanning time usually not compatible with unpredictable fetal wriggling.

\section{(Figure 3)}

\section{Brain metabolism in the normal and impaired fetal brain}

The fetal stage, the period running from the $9^{\text {th }}$ gestational week (or the end of the $11^{\text {th }}$ week of amenorrhea) to birth is characterized by critical phases of brain growth and development (ie proliferation of cells especially within the germinal matrix, development of cortical and subcortical structures, formation of sulci and gyri, migration and differentiation of neurons and glial cells, elaboration of neural networks, initiation of myelination). 
Since the publication of the first proton MRS of the human fetal brain in 1994 a limited number of studies have investigated changes in fetal brain metabolism over pregnancy, mostly during the $2^{\text {nd }}$ and $3^{\text {rd }}$ trimesters (Girard et al, 2006a; Girard et al, 2006b; Evangelou et al, 2016). Yet, these studies have contributed to unveil metabolic changes accompanying major processes in brain development as well as their timing during the fetal period. These studies have been performed with proton MRS at short TE (20 ms with STEAM or $30 \mathrm{~ms}$ with PRESS) and/or long TE (135 or $144 \mathrm{~ms}$ ) with voxels positioned in various structures (e.g. centrum semi ovale (deep white-matter), thalamus). Fetuses were considered normal when they had normal standard MRI and/or normal brain examination postnatally and normal neurological outcome depending on the clinical centers. These studies have demonstrated that NAA could be detected as early as 22 weeks of gestational age (Girard et al, 2006a) and even earlier (18 weeks of gestational age) more recently (Evangelou et al, 2016). They have also shown that NAA was increasing throughout gestation (Figure 4). NAA is expressed in neurons and oligodendrocyte progenitors in the developing brain, its production at such an early gestational age could reflect neuron differentiation with elaboration of dendrites and synapses but also the proliferation of oligodendrocytes that will be involved in myelination. Another significant change was the increase of creatine plus phosphocreatine, two compounds involved in energy metabolism, with advancing gestational age. Contradictory findings have been reported for myo-inositol, a polyol with osmoregulatory properties involved in the phosphoinositide signaling pathway, and for glutamine plus glutamate. These discrepancies may be related to the position of the voxels in different brain structures or different analytical methods. Several studies have established normative model curve fits for each metabolite that could aid detect impaired metabolic profiles. Although in utero detection of cerebral metabolic anomalies related to pathological processes is feasible, only few isolated cases have been described including intrauterine restricted growth and cytomegalovirus infection (Girard et al, 2006b). 


\section{(Figure 4)}

\section{Conclusion}

Fetal brain MRS is technically feasible and normative curves have been established from different brain regions mostly with the PRESS sequence at $1.5 \mathrm{~T}$. These data obtained from normal fetuses could aid diagnose metabolic anomalies in fetuses with abnormal MRI or at risk of brain damage. Normative curves established at $3 \mathrm{~T}$ are required since there is an increasing number of scanners operating at this magnetic field strength. One of the main challenges in MRS is data post-processing which is not easy to perform in the clinical setting without the help of spectroscopists.

\section{Summary}

Proton Magnetic Resonance Spectroscopy (MRS) is a non-invasive and quantitative method used to assess fetal brain metabolism during the $2^{\text {nd }}$ and $3^{\text {rd }}$ trimesters of gestation. Fetal brain MRS is performed in specialized centers using clinical MR systems operating at 1.5 or $3 \mathrm{~T}$. Single voxel spectroscopy (SVS) with the Point RESolved Spectroscopy (PRESS) sequence (spin echo sequence) is currently the dominant method in this field. The major metabolic changes observed in the normal fetal brain are the increase of neuronal marker $N$-acetylaspartate and of creatine and phosphocreatine with advancing gestational age. Metabolic anomalies have been reported in cases of intrauterine restricted growth and cytomegalovirus infection.

\section{Cross references}




\section{Legend to the figures}

Figure 1: Chemical structures of the major brain metabolites detected by in vivo ${ }^{1} \mathrm{H}-\mathrm{MRS}$

Figure 2: Simplified diagram of the PRESS sequence. Gx, Gy and Gz: slice-selecting gradients, RF: radiofrequency, SE: spin echo, TE: time of echo, VOI: volume of interest.

Figure 3: Example of data processing using CSIAPY, a software under Python using a time-domain quantitation algorithm based on QUEST and a simulated metabolic database. The spectrum in black is the original one, whereas the one in green is the fitted spectrum including simulated signals from metabolites and macromolecules. The red spectrum represents the contribution of macromolecule signals to the fitted spectrum. The residue is the result of the difference of the original spectrum and the fitted one.

\section{Figure 4: Typical fetal brain proton spectra acquired with the PRESS sequence at 3T}

A: Anatomical MRI showing the position of the voxel within the brain. The voxel is located in the centrum semiovale (deep white matter). B and C: spectra from a normal fetus aged 36 weeks of gestational age (GA). D and E: spectra from a normal fetus aged 29 weeks of gestational age (GA). The spectra show an increase of NAA and total creatine ( $\mathrm{tCr}$ ) with increasing GA. Abbreviations: Asp: aspartate, $\mathrm{tCr}$ : total creatine (creatine+phosphocreatine),

tCho: total Choline (phosphocholine+ glycerophosphocholine), Glx: glutamine + glutamate, NAA: $N$-acetylaspartate, $m y o$-Ins: $m y o$-inositol. 


\section{References}

- de Graaf R (2007) In vivo NMR spectroscopy, Principles and techniques. Wiley.

- Evangelou IE, du Plessis AJ, Vezina G et al (2016) Elucidating metabolic maturation in the healthy fetal brain using 1H-MR Spectroscopy. AJNR Am J Neuroradiol 37: 360366.

- Girard N, Fogliarini C, Viola A et al (2006a) MRS of normal and impaired fetal brain development. Eur J Radiol 57: 217-225.

- Girard N, Gouny SC, Viola A et al (2006b) Assessment of normal fetal brain maturation in utero by proton magnetic resonance spectroscopy. Magn Reson Med 56: 768-775.

- Heerschap A, van den Berg PP (1994) Proton magnetic resonance spectroscopy of human fetal brain. Am J Obstet Gynecol 170: 1150-1151. 
Figure 1

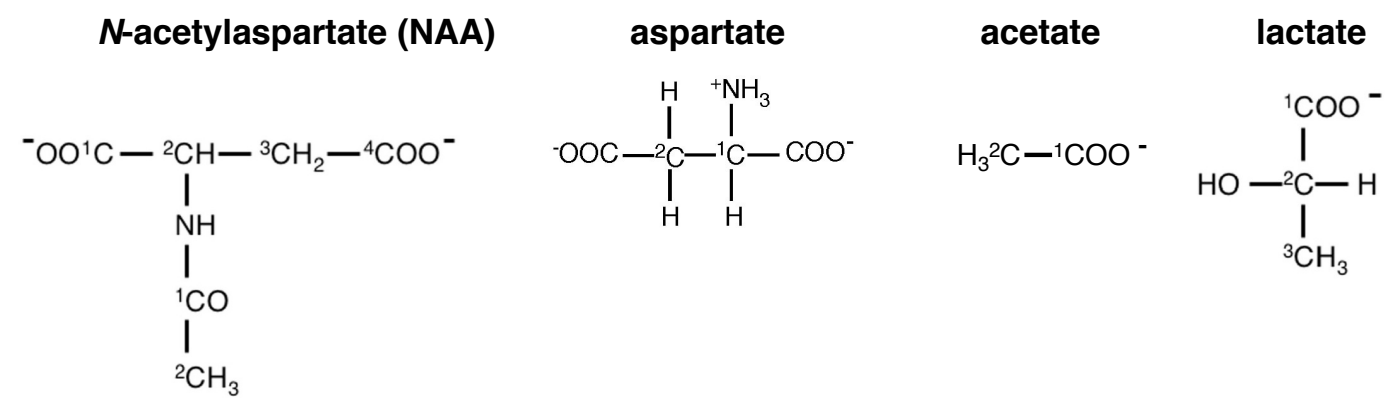

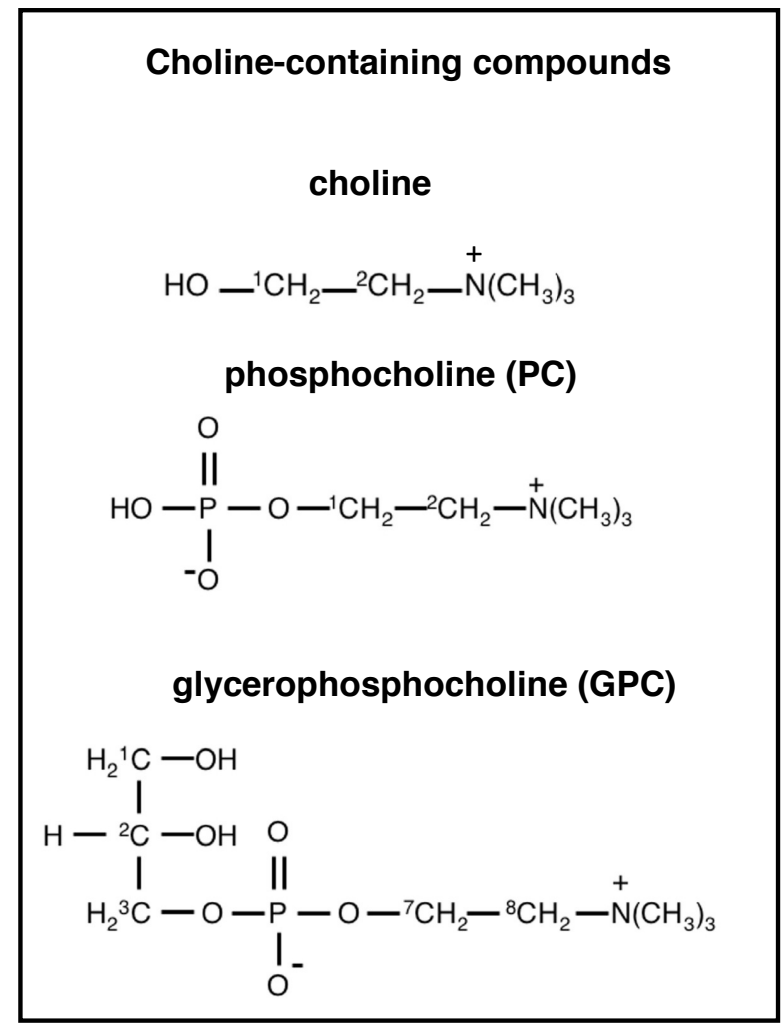

taurine<smiles>[NH3+]CCC[SeH]</smiles>

Polyols scyllo-inositol

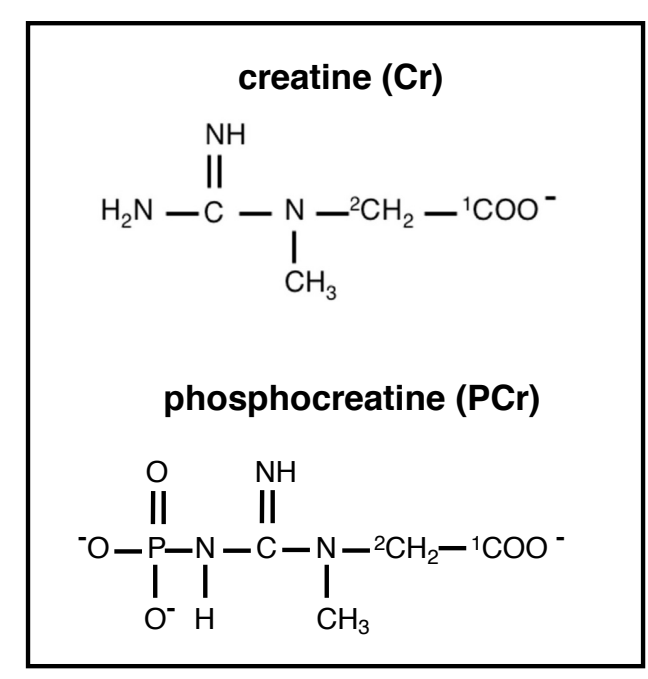

glutamine

${ }^{-} \mathrm{OO}^{1} \mathrm{C}-{ }_{+}^{{ }^{2} \mathrm{CH}}-{ }^{3} \mathrm{CH}_{3}-{ }^{4} \mathrm{CH}_{2}-{ }^{5} \mathrm{CO}-\mathrm{NH}_{2}$

glutamate<smiles>NC(CCCC(=O)[O-])C(=O)[O-]</smiles>

$\gamma$-aminobutyrate (GABA)

$\stackrel{+}{\mathrm{N}} \mathrm{H}_{3}-{ }^{4} \mathrm{CH}_{2}-{ }^{3} \mathrm{CH}_{2}-{ }^{2} \mathrm{CH}_{2}-{ }^{1} \mathrm{COO}-$ 
Figure 2

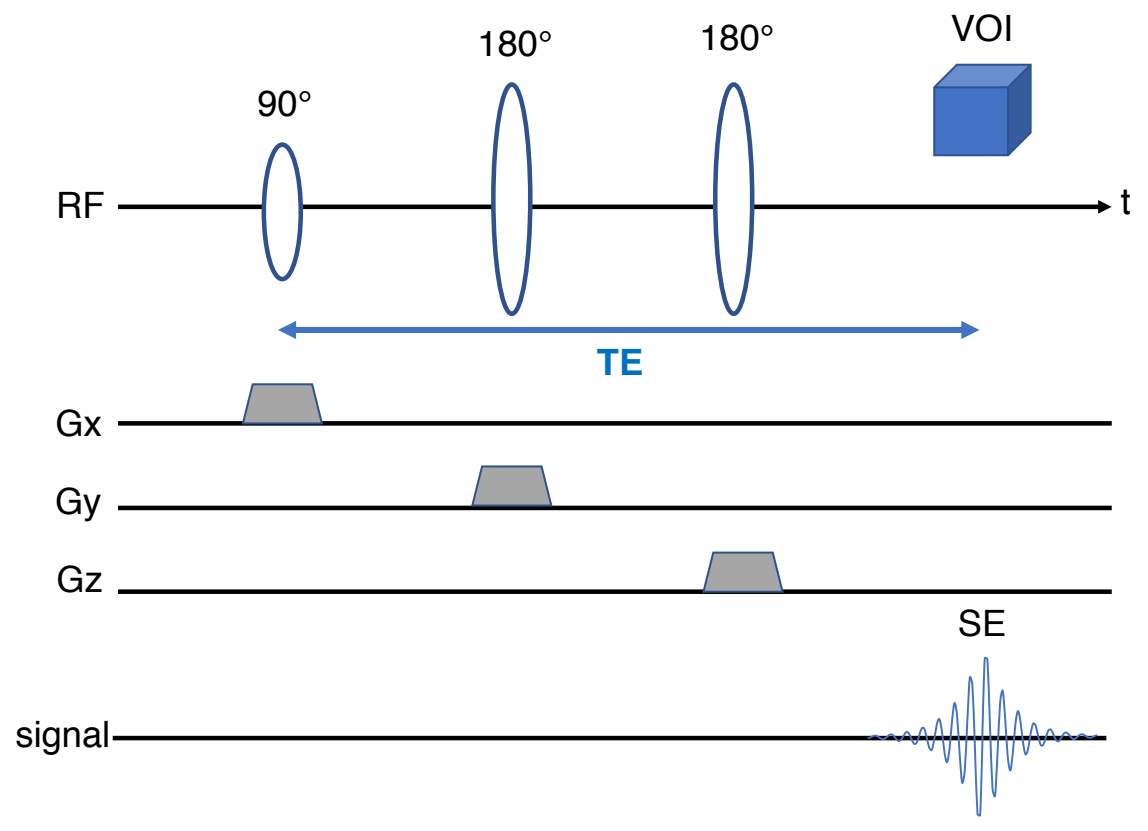


Figure 3

\section{Fitted spectrum}

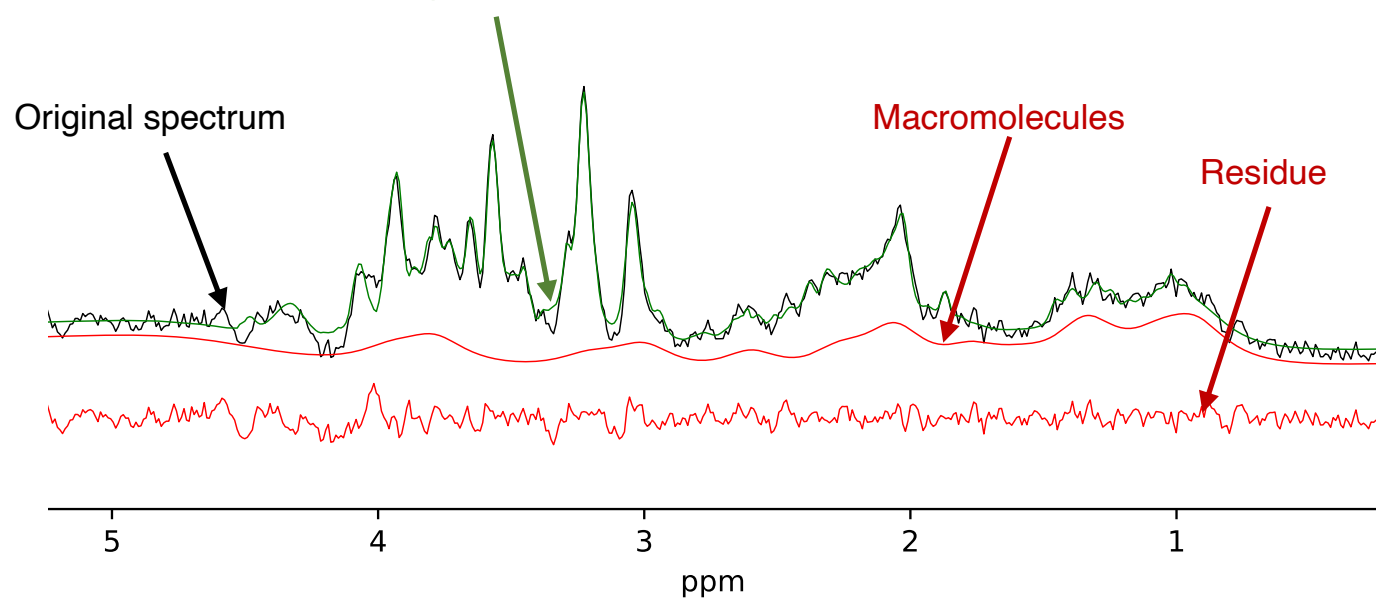


Figure 4
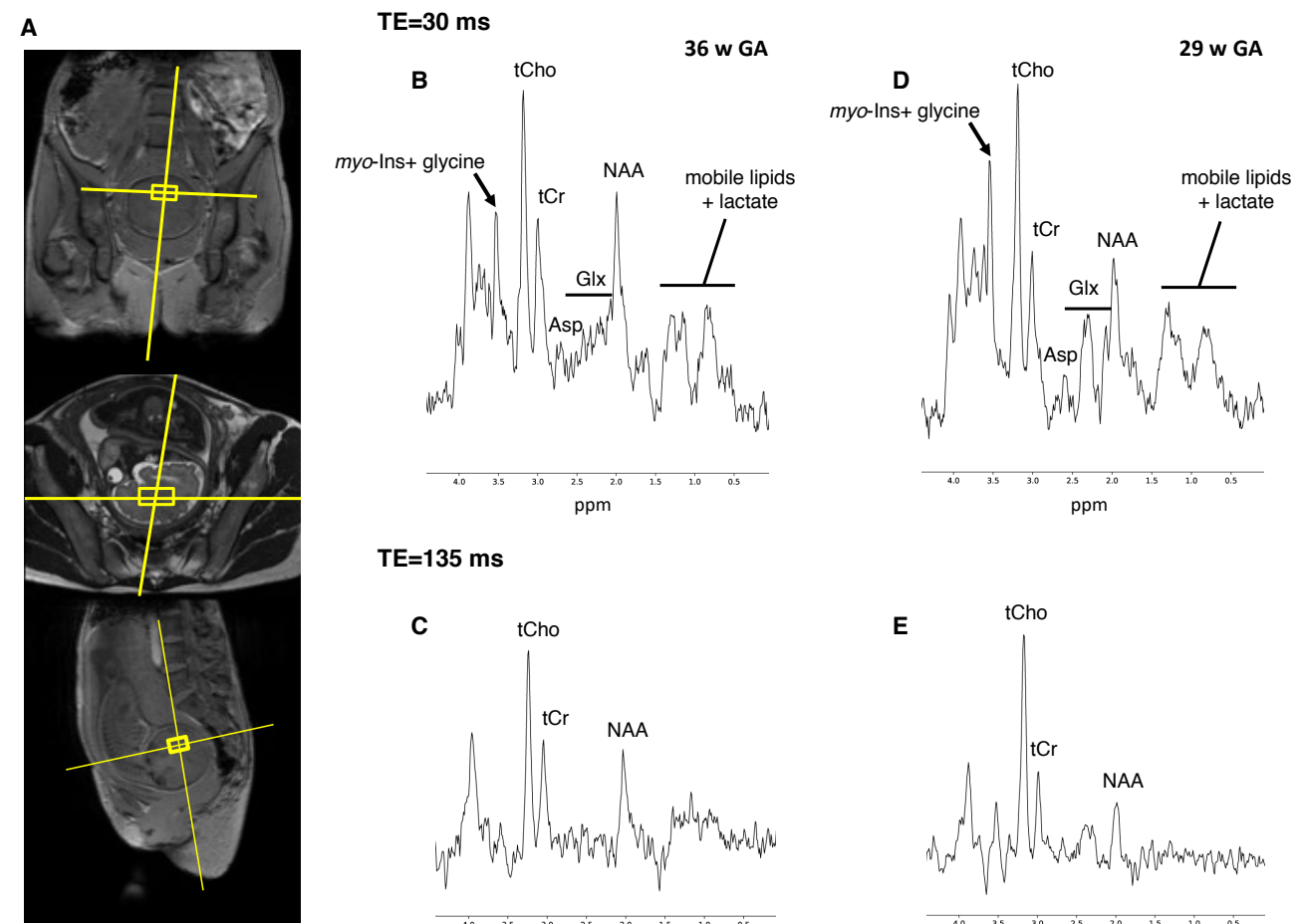

$T E=135 \mathrm{~ms}$
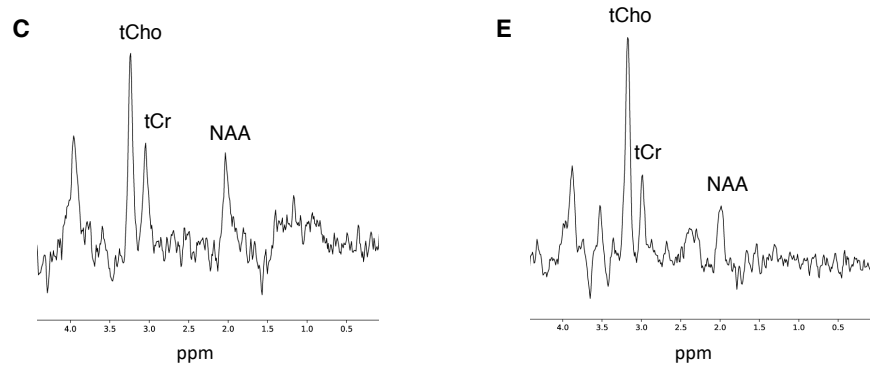